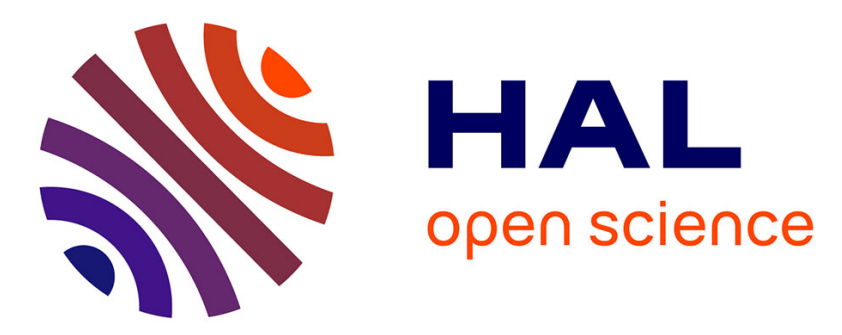

\title{
Object-based Representation and Classification of Spatial Structures and Relations
}

Florence Le Ber, Amedeo Napoli

\section{To cite this version:}

Florence Le Ber, Amedeo Napoli. Object-based Representation and Classification of Spatial Structures and Relations. International Conference on Tools with Artificial Intelligence - ICTAI'2002, Nov 2002, Washington DC, Etats-Unis d'Amerique, 8 p. inria-00107568

\section{HAL Id: inria-00107568 \\ https://hal.inria.fr/inria-00107568}

Submitted on 19 Oct 2006

HAL is a multi-disciplinary open access archive for the deposit and dissemination of scientific research documents, whether they are published or not. The documents may come from teaching and research institutions in France or abroad, or from public or private research centers.
L'archive ouverte pluridisciplinaire HAL, est destinée au dépôt et à la diffusion de documents scientifiques de niveau recherche, publiés ou non, émanant des établissements d'enseignement et de recherche français ou étrangers, des laboratoires publics ou privés. 


\title{
Object-based Representation and Classification of Spatial Structures and Relations
}

\author{
Florence Le Ber and Amedeo Napoli \\ LORIA - UMR 7503, B.P. 239, 54506 Vandœuvre-lès-Nancy Cedex, France \\ \{leber,napoli\}@loria.fr
}

\begin{abstract}
This paper is concerned with the representation and the classification of spatial relations and structures in an object-based knowledge representation system. In this system, spatial structures are defined as sets of spatial entities connected with topological relations. Relations are represented by objects with their own properties. We propose to define two types of properties: the first ones are concerned with relations as concepts while the second are concerned with relations as links between concepts. In order to represent the second type of properties, we have defined facets that are inspired from the constructors of description logics. We describe these facets and how they are used for classifying spatial structures and relations on land-use maps. The links between the present work and related work in description logics are also discussed.
\end{abstract}

\section{Introduction}

This paper focuses on the representation and classification of spatial relations and structures in an objectbased knowledge representation system (or ObKR system). Our proposition is to extend the representation capabilities of ObKR systems in order to represent spatial relations as "first-class citizens", i.e., classes with their own properties. The underlying assumption is that spatial relations, like part-whole relations, cannot be simply represented by ordinary attributes and must be represented as semantic constructs $[1,24]$. The ideas presented here have been implemented for designing a knowledge-based system, named LoLA (Lorraine Landscape Analysis), for agricultural landscape analysis on land-use maps. Furthermore our proposition can be taken as a general basis for representing and manipulating relations in an ObKR system.

The purpose of the LOLA system is to analyze land-use maps based on satellite images in order to recognize spatial structures (models of landscape) that have been defined by agronomists [13]. The results are used for global diagnoses and forecasts on agriculture with regard to the environment. In our framework, the problem of recognizing spatial structures on images is considered as a classification problem, where the spatial structures correspond to classes, and the regions and sets of regions of the images correspond to individuals to be classified, with respect to classes (see Fig. 1). Actually, spatial structures are described as sets of spatial entities (or atoms) connected by spatial relations. For example, a partial description of the structure of a village territory (denoted by $x$ ) is given below in natural language:

1. "all fields belonging to $x$ are large fields"

2. "all state forests are disconnected from $x "$

3 . " $x$ is bordered with at least one meadow"

4. " $x$ contains at most one field on the borders"

This example shows how the agronomists describe spatial structures. They use various quantifiers such as: all, none, at least, at most, and spatial relations that can be specialized, e.g. contain and contain on the borders. Spatial entities can also be specialized: for example, forests into state forests, fields into large fields or small fields. Spatial structures are specialized accordingly. These two specialization mechanisms must be taken into account in the classification process.

In our framework, the spatial structures are represented by classes and the image regions by instances within an ObKR system. The recognition of spatial structures on images relies on instance classification. The choice of an ObKR system relies on a number of reasons: this kind of system allows both computation -to check relations and quantification on the images-, knowledge representation and classification-based reasoning. Moreover, we propose an original extension of the representation capabilities of a classical ObKR system by considering spatial relations as "first-class citizens", i.e., classes with their own properties and behavior. Thus, relations are represented by classes having attributes and organized within a class hierarchy, actually a lattice. Furthermore, the classical classification 


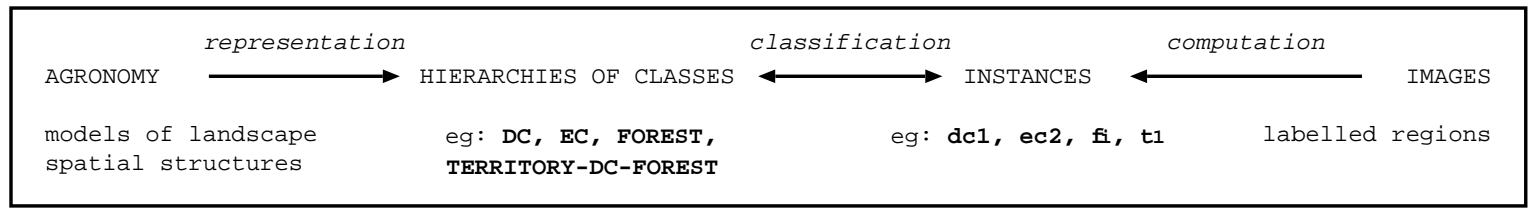

\section{Figure 1. The recognition of spatial structures can be seen as a classification problem in an ObKR system: the models of the agronomists are represented by classes, the regions and relations com- puted on the images are represented by instances.}

mechanism has been adapted, to take into account two hierarchies: the hierarchy of spatial structures and the hierarchy of spatial relations. This gives rise to a bi-dimensional classification mechanism: the classification of an image region, represented by an instance, say $\mathrm{x}$, requires the classification of the image regions related to $\mathrm{x}$, and the classification of the spatial relations linking these regions to $\mathrm{x}$.

Our proposition, inspired from research works in description logics (DL), is original since the reification of relations has never been implemented in ObKR systems for qualitative spatial reasoning on images. Furthermore, this proposition addresses an important question in qualitative spatial reasoning, that is to link qualitative models with quantitative data, and to implement these qualitative models for real-world applications [29].

This paper is organized as follows. Section 2 introduces the spatial relations we have used, namely topological relations. Sections 3 and 4 describe our choices for representing spatial relations and structures. Section 5 describes the classification mechanism. Section 6 is a discussion on the present work and related work. Section 7 concludes the paper.

\section{Topological Relations}

The agronomists use many qualitative spatial relations to describe landscape spatial structures. They use mainly topological relations, but also distance, and orientation relations. We have focused on the topological relations because they are binary relations that have been formalized in an exhaustive manner. Furthermore, they allow to describe most of the landscape models. Actually, our framework relies on the Region Connection Calculus RCC-8, that is based on eight base relations [23]:

- $E Q(x, y):$ : $x$ is identical with $y$ ”,

- $N T P P(x, y)$ : “ $x$ is a non tangential proper part of $y$ ',

- $T P P(x, y):$ : $x$ is a tangential proper part of $y$ ',

- $N T P P^{-1}(x, y)$ : “x non tangentially contains as a proper part $y$,'

- $T P P^{-1}(x, y)$ : “x tangentially contains as a proper part y”,
- $P O(x, y)$ : “x partially overlaps y”,

- $E C(x, y)$ : “x is externally connected with y",

- $D C(x, y)$ : “ $x$ is disconnected from $y$ ”.

Topological relations can be computed on images thanks to set operations as shown in [7]. Such operations rely on the interiors and boundaries of the regions, which leads to specific problems in discrete spaces. We have proposed a specific solution for land-use maps [14]. Let $\Delta$ be the set of the regions of an image, that are regular, with no hole, closed, and not necessarily connected. A region $x \in \Delta$ is made of two sets of pixels, the interior $\left(x^{\circ}\right)$ and the boundary $(\partial x)$. We consider four operations between two regions $x$ and $y$ : the intersection of the interiors, $x^{\circ} \cap y^{\circ}$, the intersection of the boundaries, $\partial x \cap \partial y$, the differences of the interiors, $x^{\circ}-y^{\circ}, y^{\circ}-x^{\circ}$. The result of these operations may be empty or not empty. Eight conditions are defined accordingly and denoted as follows:

- $P(x, y): x^{\circ}-y^{\circ}=\emptyset$;

- $P^{-1}(x, y): y^{\circ}-x^{\circ}=\emptyset$;

- $D x(x, y): x^{\circ}-y^{\circ} \neq \emptyset$;

- $D y(x, y): y^{\circ}-x^{\circ} \neq \emptyset$;

- $O(x, y): x^{\circ} \cap y^{\circ} \neq \emptyset$

- $\operatorname{DR}(x, y): x^{\circ} \cap y^{\circ}=\emptyset$;

- $A(x, y): \partial x \cap \partial y \neq \emptyset$;

- $N A(x, y): \partial x \cap \partial y=\emptyset$.

The conjunctions of these eight conditions are equivalent to the eight base relations of RCC-8 (Tab. 1). Then, computing a relation on the image is the same operation as verifying a set of conditions. For example, the relation $E C$ is associated with the set of conditions: $\mathcal{C}=$ $\{D R, A, D y, D x\}$. Based on this relationship between the RCC-8 base relations and the eight conditions, a Galois lattice - also called formal concept lattice [8] — has been defined $[14,15]$. An element $E$ of the lattice is an ordered pair $(\mathcal{C}, \mathcal{R})$, where $\mathcal{C}$ is a subset of conditions and $\mathcal{R}$ is a subset of base relations such that:

$$
\forall(x, y) \in \Delta^{2}, \bigvee_{r \in \mathcal{R}} r(x, y) \leftrightarrow \bigwedge_{c \in \mathcal{C}} c(x, y)
$$




$\begin{array}{lll}E Q & \leftrightarrow & P \wedge P^{-1} \wedge A \wedge O \\ N T P P & \leftrightarrow & P \wedge D y \wedge N A \wedge O \\ T P P & \leftrightarrow & P \wedge D y \wedge A \wedge O \\ N T P P^{-1} & \leftrightarrow & P^{-1} \wedge D x \wedge N A \wedge O \\ T P P^{-1} & \leftrightarrow & P^{-1} \wedge D x \wedge A \wedge O \\ P O & \leftrightarrow & O \wedge D x \wedge D y \wedge A \\ E C & \leftrightarrow & D R \wedge A \wedge D y \wedge D x \\ D C & \leftrightarrow & D R \wedge N A \wedge D y \wedge D x\end{array}$

Table 1. Equivalences between the RCC-8 relations and the conjunctions of conditions.

Thus, each relation that is represented in the lattice can be checked on the image using conditions. Furthermore the partial ordering $\sqsubseteq$ in the lattice is equivalent to the logical implication on the relations:

$$
\begin{aligned}
\left(\mathcal{C}_{1}, \mathcal{R}_{1}\right) & \sqsubseteq\left(\mathcal{C}_{2}, \mathcal{R}_{2}\right) \\
\forall(x, y) \in \Delta^{2}, \bigvee_{r \in \mathcal{R}_{1}} r(x, y) \rightarrow & \bigvee_{r \in \mathcal{R}_{2}} r(x, y)
\end{aligned}
$$

This lattice structure provides other interesting properties for reasoning purposes. In particular, the greatest lower bound (denoted $g l b$ ) of two elements is equivalent to the conjunction of the two corresponding relations or conditions. Thus, if two relations have been checked separately for two regions, one can deduce directly what relation holds between the two regions. Moreover, the composition of two relations can be deduced from the Galois lattice and from the composition table of the RCC-8 base relations as it is done in [22].

A complete analysis of the properties of the Galois lattice and a comparison with other lattices is given in [14, 15].

\section{The Representation of Topological Rela- tions in an ObKR System}

Up to now there is no satisfying reification of relations in ObKR systems [1]. Moreover, the relation notion can be considered as being in opposition with the object notion. The specialization and instantiation relations are commonly taken into account, as well as composition in some ObKR systems, whereas many other relations, such as spatial or temporal relations, may be useful in many applications.

Actually, the purpose of our work is to propose an objectbased environment for the representation and manipulation of topological relations. We have used the ObKR system Y3 that includes a frame-based language YAFOOL and an interface YAFEN [6]. In YAFOOL all objects -classes and instances- are represented by frames; frames are composed of slots, representing both attributes and methods [20]. Attributes can be characterized by declarative and procedural facets: the former are used to represent the range and the value of the attribute while the latter are used to specify local behaviors. Methods associate a behavior to a frame and are activated by message sending. Attributes, facets and methods are objects. Binary relations are special kinds of attributes characterized by the fact that their range is a user-defined class. The classification and inheritance mechanisms are based on attribute unification. When classifying an instance into a reference class, the system checks whether the (attribute, range) or (attribute, value) pairs in the instance are conform to the pairs in the class; if this is the case, the instance can be classified into the class.

Based on Y3 capabilities, topological relations are represented by classes and organized within a hierarchy according to the lattice ordering defined in Section 2. Every relation class (denoted by TR-class in the following) is a specialization of the generic class SPATIAL-RELATION that introduces the attributes and the methods (in the objectoriented sense) common to all TR-classes (see Fig. 2). For example the TR-classes EC, TPP ${ }^{-1}, \mathrm{DC}$, respectively representing the relations $E C, T P P^{-1}, D C$, are subclasses of SPATIAL-RELATION and inherit its properties (see Fig. 3 \& 4). Besides, the TR-classes A, 0, etc., respectively represent the conditions $A, O$, etc.

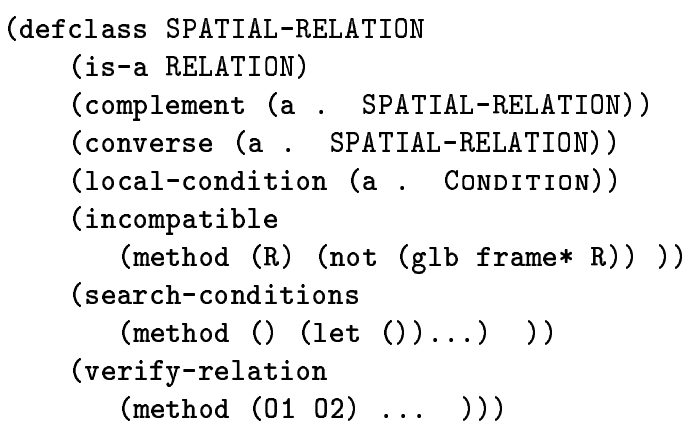

Figure 2. The SPATIAL-RELATION class - specialization of the special class RELATION coded in $\mathrm{Y} 3$.

The attributes of the class SPATIAL-RELATION describe the properties of the relations. The value of the complement attribute of a TR-class $R$ is the TR-class $R^{c}$ such that the relations $R$ and $R^{c}$ are both exhaustive and disjoint for any region pair of $\Delta$. The converse attribute of a TR-class $\mathrm{R}$ is the TR-class $\mathrm{R}^{-1}$ such that $R(x, y)$ holds whenever $R^{-1}(y, x)$ holds. The value of the attribute local-condition is the set of conditions being equivalent to the relation $R$ (only defined in certain TR-classes: Dx, Dy, $\mathrm{DR}, \mathrm{O}, \mathrm{A}, \mathrm{NA}, \mathrm{P}, \mathrm{P}^{-1}$ ) [14].

There are three main methods exploiting the lattice struc- 
ture of the TR-classes to find out:

- the greatest lower bound of two relations.

- the set of conditions associated with a relation.

- the relation associated with a set of conditions.

The method incompatible checks whether two relations are disjoint $\left(g l b\left(\mathrm{R}_{1}, \mathrm{R}_{2}\right)=\perp\right)$ or not. The verify-relation method checks whether the relation $R$ exists between two regions of an image. It uses the search-conditions method that returns the set of conditions $\mathcal{C}$ associated with the relation. If the verify-relation method succeeds, it creates an instance of the relation class. If it fails (one of the conditions of $\mathcal{C}$ is false), it searches for the relation that is associated with the set of conditions it has computed and creates an instance of this relation class. For example, suppose that the relation $E C(x, y)$ has to be checked, $\mathcal{C}=$ $\{D R, A, D y, D x\}$, and that $A(x, y)$ is false; then $N A(x, y)$ is true, $\{D R, N A, D y, D x\}$ is verified and an instance of $\mathrm{DC}$ is created (see Tab. 1).

\section{Representation of Spatial Structures}

ObKR systems share many characteristics with description logics. In DL, concepts are used to represent classes of individuals while roles are used to represent binary relations between classes [5]. The description of a concept is composed of roles with which are associated constructors introducing restrictions on the roles, e.g. range, cardinality, universal and existential quantification. Relying on these characteristics of description logics, we have extended the Y3 system in order to represent spatial structures and relation quantification.

\subsection{Quantifiers and Facets}

As introduced in Section 1, the spatial structures are described with quantifiers. Four main quantifiers have been used in our application. We give below the four sentences introduced as an example in Section 1 translated into logical formulas, using the RCC-8 relations, and then we make the correspondence with the standard quantifiers of description logics ( $x$ is supposed to be an existentially quantified variable, denoting the spatial region under description).

\section{1. "all fields belonging to $x$ are large fields":} $\forall y($ field $(y) \wedge \operatorname{contain}(x, y) \rightarrow$ large-field $(y))$

This first implication can be represented in DL using the constructors all and range:

$x=$ (all (range contain field) large-field).
2. "all state forests are disconnected from $x "$ : $\forall y$ (state-forest $(y) \rightarrow$ disconnected $(x, y))$

This second implication is different from the previous one since its conclusion involves a relation. Thus the all constructor cannot be used in a classical way. An alternative is to use the converse implication $\neg B \rightarrow$ $\neg A$ rather than $A \rightarrow B$. The converse implication can be represented in DL, using the negation on roles [17]: $x=($ all (not disconnect) (not state-forest)).

3. " $x$ is bordered with at least one meadow": $\operatorname{card}\{y \mid \operatorname{meadow}(y) \wedge$ externally-connected $(x, y)\} \geq 1$

This third formula can be represented in DL using the qualified number restriction c-atleast [28]:

$\mathrm{x}=$ (c-atleast 1 externally-connected meadow).

4. " $x$ contains at most one field on the borders": $\operatorname{card}\{y \mid \operatorname{field}(y) \wedge$ tangentially-contain $(x, y)\} \leq 1$

This formula can be represented in DL using the qualified number restriction c-atmost:

$x=$ (c-atmost 1 tangentially-contain field).

The quantifiers all, c-atleast, c-atmost, were not available in the current version of Y3. Thus, relying on the models of the corresponding constructors in description logics, we have implemented facets that can be associated with relations and that represent these quantifiers. According to our specific needs, as exemplified by the four sentences above, we have designed four facets, namely super-range, all-role, c-atleast and c-atmost, that are detailed hereafter.

\subsection{Spatial Structures}

The spatial structures are described as sets of spatial entities connected with spatial relations. The spatial entities are represented by primitive classes, named EN-classes in the following, e.g. FIELD, BARLEY, FOREST. The EN-classes are combined with TR-classes to define the ST-classes that represent the spatial structures, e.g. TERRITORY ${ }^{1}$ (see Fig. 3). The attributes of ST-classes are particular relations, instances of the TR-classes. Furthermore, these attributes are characterized by several facets: their ranges are EN-classes or ST-classes, and the facets super-range, all-role, c-atleast and c-atmost are used for quantification purpose.

Examples of spatial structures are given in Fig. 4. The FIELD-EC-FOREST class represents the fields externally connected to exactly one forest; it is defined with the attribute $g-e c-f$ and the facet c-exactly that is the conjunction of c-atleast and c-atmost.

\footnotetext{
${ }^{1}$ Territory stands for village territory.
} 


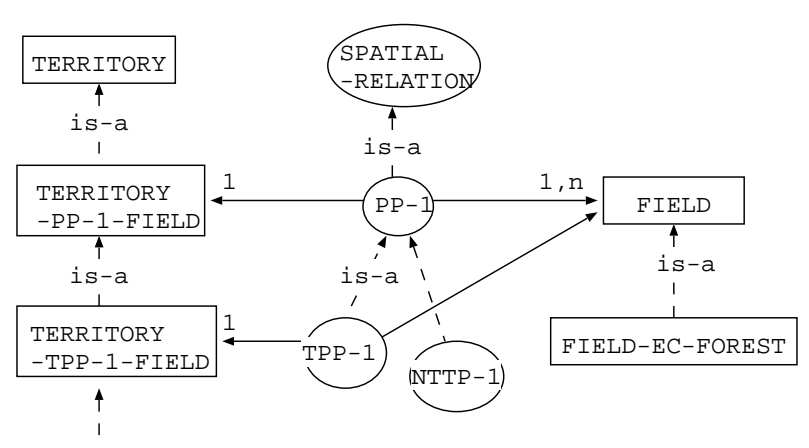

Figure 3. Linking hierarchies of ST-classes (TERRITORY) and EN-classes (FIELD) via the TRclasses $\left(\mathrm{PP}^{-1}, \mathrm{TPP}^{-1}\right)$.

The TERRITORY-PP ${ }^{-1}$-FIELD class represents village territories that contain at least one field. The TERRITORY-TPP ${ }^{-1}$-FIELD class represents village territories containing at least one field on their borders. The TERRITORY-PP ${ }^{-1}$-FIELD-EC-FOREST class represents the territories whose fields are all instances of the class FIELD-EC-FOREST; it is defined with the facet super-range. The TERRITORY-DC-FOREST class represents the territories that are disconnected from all forests; it is defined with the attribute $t-d c-f$ and the facet all-role that corresponds to the combination of the constructor all with the negation on roles.

It can be noticed that facets corresponding to other specific needs could have been defined as well, as far as the complexity of reasoning is reasonable, as discussed below.

Finally, the ST-classes can be specialized in three different ways (Fig. 4):

- Adding a relation: the FIELD class is specialized in FIELD-EC-FOREST or FIELD-DC-FOREST.

- Specializing a relation: the TERRITORY-PP ${ }^{-1}$-FIELD class can be specialized in TERRITORY-TPP ${ }^{-1}-$ -FIELD.

- Specializing the range of a relation: TERRITORY$-\mathrm{PP}^{-1}$-FIELD is specialized in TERRITORY-PP ${ }^{-1}$ -FIELD-EC-FOREST.

An EN-class or a ST-class, say C, may invoke a main classification method, named recognize. The role of this method is to check whether a region or a set of regions of an image can be classified with respect to the class $\mathrm{C}$. If the test succeeds then an instance of $\mathrm{C}$ is created (details are given in Section 5).

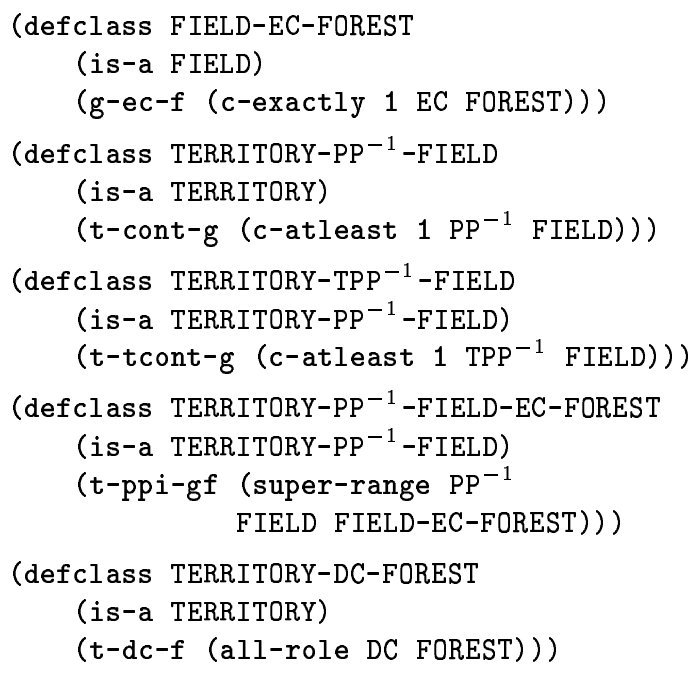

Figure 4. ST-classes coded in Y3. The attributes (e.g. $g-e c-f$ ) are instances of TR-classes (e.g. EC) and are characterized by facets representing quantifiers (e.g. c-exactly). Their range is an EN-class or a ST-class (e.g. FOREST).

\subsection{Interpretation and Complexity}

As in DL frameworks, we call an interpretation the pair $\mathcal{I}=\left(\Delta,{ }^{\mathcal{I}}\right)$ where $\Delta$ is a set of image regions, as introduced in Section 2, called the interpretation domain, and.$^{\mathcal{I}}$ is an interpretation function, mapping a class to a subset of $\Delta$ and an attribute to a subset of $\Delta \times \Delta$. The interpretation of a class relies on the interpretation of its attributes. Methods are not taken into account since they have no sideeffects: actually they are comparable to the test functions of CLASSIC [4]. Furthermore, the interpretation of an attribute att depends on its facets. In the following, we give the interpretation of the four facets introduced above for representing spatial structures, where att is an instance of the $R$ relation class, $\mathrm{C}$ is the range of att, and $\mathrm{SC}$ is a class more general than $\mathrm{C}$ :

$$
\begin{aligned}
& (\mathrm{c} \text {-atleast n R C })^{\mathcal{I}}= \\
& \left\{x \in \Delta \mid \operatorname{card}\left\{y \in \Delta \mid(x, y) \in \mathrm{R}^{\mathcal{I}} \wedge y \in \mathrm{C}^{\mathcal{I}}\right\} \geq n\right\} \\
& (\mathrm{c} \text {-atmost n R C) })^{\mathcal{I}}= \\
& \left\{x \in \Delta \mid \operatorname{card}\left\{y \in \Delta \mid(x, y) \in \mathrm{R}^{\mathcal{I}} \wedge y \in \mathrm{C}^{\mathcal{I}}\right\} \leq n\right\} \\
& (\text { all-role R C })^{\mathcal{I}}= \\
& \left\{x \in \Delta \mid \forall y \in \Delta, y \in \mathrm{C}^{\mathcal{I}} \rightarrow(x, y) \in \mathrm{R}^{\mathcal{I}}\right\} \\
& (\text { super-range R SC C) })^{\mathcal{I}}= \\
& \left\{x \in \Delta \mid \forall y \in \Delta,\left(y \in \operatorname{SC}^{\mathcal{I}} \wedge(x, y) \in \mathrm{R}^{\mathcal{I}}\right) \rightarrow y \in \mathrm{C}^{\mathcal{I}}\right\}
\end{aligned}
$$

It is interesting to compare this extension of Y3, including the four facets, with the DL family $\mathcal{A L}$ [5]. The three 
facets, c-atleast, c-atmost, and super-range, require the constructor range which itself implicitly requires the negation and the disjunction of defined concepts [27]. Thus, the complexity of the extension of $\mathrm{Y} 3$ is similar to that of $\mathcal{A L C}$ : detecting the subsumption relations is PSPACEcomplete. Furthermore, the fourth facet, all-role, introduces the negation on roles, and it is proved in [17] that this extension in $\mathcal{A L}$ makes the satisfiability of concepts ExpTime-complete. Finally, the complexity of reasoning in this extension of $\mathrm{Y} 3$ is comparable to the current versions of description logics such as RACE [10] or SHIQ [11, 12].

\section{Spatial Structure Classification}

In description logics, concepts are organized within a hierarchy according to a subsumption relation. Reasoning is based on concept classification and instance recognition. Concept classification is used to insert a new defined concept in the concept hierarchy by searching for its most specific subsumers and its most general subsumees. Instance recognition is aimed at searching for the most specific concepts of which an individual may be an instance [26]. The search in the hierarchy is usually performed top-down and depth-first.

The classification and recognition processes in our framework are based on these same principles. When classifying an instance $\mathrm{x}$ of a spatial structure, the classification mechanism has to check both the domain and the range of the attributes of $\mathrm{x}$. The classification process is progressive: the attributes of $\mathrm{x}$ are checked step by step, only when needed. Thus, instances of relations are created only when needed, in order to economize space as required in [18]. The classification process is performed top-down and depth-first, and goes on down the hierarchy of spatial structures until all classes have been checked. The general method recognize $(\mathrm{C}, \mathrm{i})$, where $\mathrm{C}$ is a spatial structure class, and $i$ is an instance, is described in Algorithm 1.

The system LoLA has been implemented and used with land-use maps of the Lorraine region (East of France). The instances represent the image regions, that are sets of pixels with the same label. Each label corresponds to a particular land-use category (forest, meadow, corn, barley, buildings, etc.) or to a village territory [2].

The system first recognizes a village territory region on an image thanks to its label. This region is represented as an instance, say $t_{1}$, of the corresponding class, TERRITORY. The system tries then to classify the instance $t_{1}$ into a more specific class, for example the TERRITORY-DC-FOREST class. It invokes therefore the recognize method of this last class, that works as follows (see Algorithm 1). It checks whether the instance $t_{1}$ matches the attributes of TERRITORY-DC-FOREST, i.e., $t-d c-f=(a l l-r o l e$ DC FOREST) (see Fig. 4). Thus

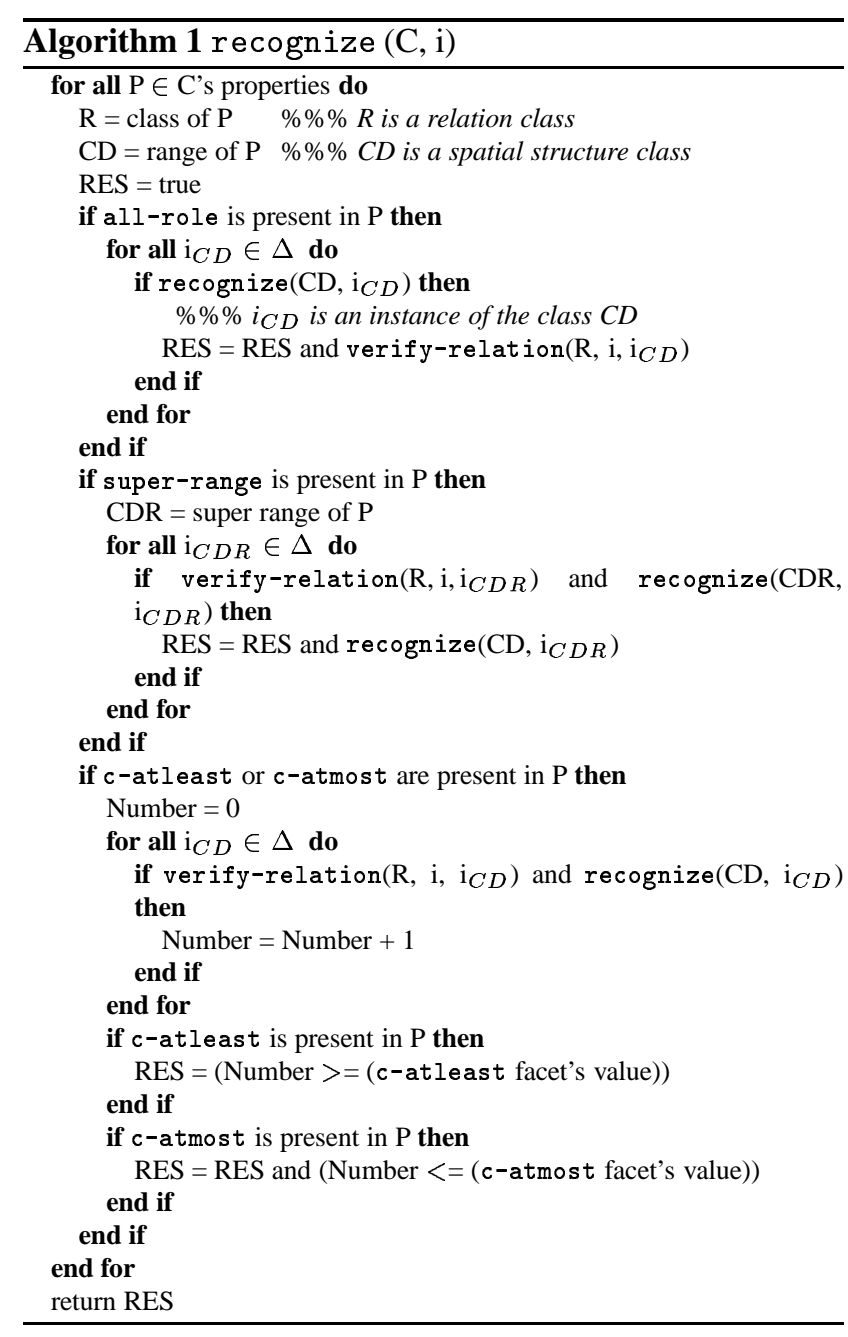

it looks on the image for forest regions, say $\mathbf{f}_{i}$, in the neighborhood of $t_{1}$ (calling the recognize method of the FOREST class), and computes the topological relation between $t_{1}$ and each forest region $f_{i}$ (using the verify-relation method of the class DC). If $D C\left(\mathrm{f}_{i}, \mathrm{t}_{1}\right)$ holds for all forest regions $f_{i}$, then an instance $\mathrm{dc}_{1}$ of DC is created that links $t_{1}$ to all the $f_{i}$, and the instance $t_{1}$ is classified into the TERRITORY-DC-FOREST class. On the contrary, if there is a forest region $\mathrm{f}_{j}$ such that $D C\left(\mathrm{f}_{j}, \mathrm{t}_{1}\right)$ does not hold, then the system infers the relation existing between $\mathbf{f}_{j}$ and $\mathrm{t}_{1}$ (e.g. $E C\left(\mathbf{f}_{j}, \mathrm{t}_{1}\right)$ ), and tries to classify $t_{1}$ into another subclass of TERRITORY (e.g. TERRITORY-EC-FOREST).

Finally, the result of the analysis is a collection of classes of which the territory is an instance. Two territories that are instances of the same classes are supposed to share the same spatial structure and thus the same agricultural behavior. According to these results, the territories of an image are grouped into regions whose maps are drawn and analyzed by the agronomists. The first results of the LoLA 
system are more deeply detailed in [16]. They are very positive and appeal for further studies and investigations both in landscape model representation and in reasoning with topological relations.

\section{Related Work}

Our approach presents some similarities with the one used in the VEIL system for the recognition process of objects in an image [25]: the domain model is described within hierarchies of concepts embedded in the LOOM system [19]. The LOOM classifier is used to classify the image objects into concepts according to the available information. The VEIL system combines both declarative knowledge and computational methods.

As in the VEIL system, we have chosen to use an ObKR system since we needed both computation and representation capabilities. In order to represent spatial structures and relations, we have improved the $\mathrm{Y} 3$ system in a number of directions. First, we consider relations as "first-class citizens", as proposed in [24], and we have defined attributes and facets to represent the properties of relations (complement, converse, etc.) and quantifiers. Moreover, we have defined and implemented a hierarchy of relations and we have accordingly modified the classification mechanism in Y3. The attributes and the facets associated with relations are based on the specific needs of our application, but they are of general use in an ObKR system. In addition, the present work gives guidelines for extending an ObKR system for special representation purpose, based on constructions inspired from description logics.

Actually, our work can be considered as complementary to research work in description logics holding on qualitative spatial representation $[9,10]$. The authors in $[9,10]$ indeed propose to represent spatial objects and relations within the RACE system, and to extend this system to the polygon concrete domain, for taking into account spatial regions. Special modeling constructs can be used to represent topological relations as defined roles, whereas we consider relations as concepts. Spatial reasoning relies on two main operations in description logics reasoning, namely consistency checking and classification. From the application point of view, regions are represented by polygons (elements of the concrete domain), and reasoning is carried on relations between polygons, for recognizing regions with specific characteristics and answering queries to a map database. The regions considered in our work are raster regions, which makes a difference between the two approaches regarding the computational aspects, but no fundamental difference from the reasoning point of view.

The underlying reasoning mechanism is based in both cases on classification, and especially relation classification. Moreover, in [21], spatiotemporal default reasoning is intro- duced: default knowledge is represented as rules and used for completing and making more precise queries. This is still a complementary view with respect to our work. In our approach, default knowledge is represented within the classes and is used to allow classification-based reasoning on spatial relation and concepts. However, a more theoretical study of our approach has still to be carried out, as it is done in [21].

\section{Conclusion and Future Work}

The present work is concerned with the design of an ObKR system for representing and classifying spatial structures. The objective is to recognize and then analyze spatial structures on satellite images, relying on the matching of the image regions with landscape models defined by the agronomists. These models are expressed as sets of spatial entities connected with qualitative spatial relations. The matching problem can be considered, in the framework of an object-based knowledge representation system, as a problem of instance classification, where the image regions are instances and the spatial structures are classes. Our proposition consists in reifying the relations for computation and reasoning purposes. We have built two dependent hierarchies of classes, the first one representing topological relations and the second one the spatial structures. Based on the DL representation principles, we have implemented four facets to improve the representation capabilities of the Y3 system, and we have accordingly adapted the classification mechanism. The resulting framework has been used successfully to implement an application for agricultural landscape analysis.

Future works are engaged in a number of directions. First, regarding ObKR systems, the current state of relation reification lacks generality. In particular, the inheritance mechanism only recognizes the specialization of ranges, whereas the specialization of relation domains should be taken into account to provide a more general reification of relations. Sharing properties via "horizontal" relations contrasting with "vertical" relations, such as specialization and instantiation- should also be considered. Finally, regarding the application itself, improvements can be made in two main ways: adding indices to characterize spatial entities and adding spatial relations to characterize spatial structures (distance, extended topology, orientation). Representing these last relations will be more difficult than representing RCC-8 relations since they are (mostly) ternary relations. Moreover, it is necessary to find a unique logical framework to represent all these qualitative relations, as proposed in a recent work [3]. 


\section{References}

[1] A. Artale, E. Franconi, N. Guarino, and L. Pazzi. Part-whole relations in object-oriented systems: An overview. Data \& Knowledge Engineering, 20(3):347-383, 1996.

[2] J. Bachacou. Map of the land use in Lorraine based on Landsat images, crop year 1989-90. Document, 1995. Laboratoire d'Intelligence Artifi cielle et de Biométrie, INRANancy, France.

[3] B. Bennett. A Categorical Axiomatisation of Region-Based Geometry. Fundamenta Informaticae, 46:145-158, 2001.

[4] R. Brachman, D. McGuinness, P. Patel-Schneider, L. Resnick, and A. Borgida. Living with CLASSIC: When and How to Use a KL-ONE-Like Language. In J. Sowa, editor, Principles of Semantic Networks: Explorations in the Representation of Knowledge, pages 401-456. Morgan Kaufmann Publishers, 1991.

[5] F.-M. Donini, M. Lenzerini, D. Nardi, and A. Schaerf. Reasoning in description logics. In G. Brewka, editor, Principles of Knowledge Representation, pages 191-236. CSLI Publications, 1996.

[6] R. Ducournau. Y3: YAFOOL, the object oriented language, 1991. SEMA Group, France.

[7] M. J. Egenhofer and J. Sharma. Topological Relations Between Regions in $\Re^{2}$ and $Z^{2}$. In D. J. Abel and B. C. Ooi, editors, Advances in spatial databases, Third International Symposium, SSD'93, Singapore, LNCS 692, pages 316-336. Springer Verlag, 1993.

[8] B. Ganter and R. Wille. Formal Concept Analysis. Springer, Berlin, 1999.

[9] V. Haarslev, C. Lutz, and R. Möller. Foundations of Spatioterminological Reasoning with Description Logics. In Proceedings of the Sixth International Conference on Principles of Knowledge Representation and Reasoning (KR'98), Trento, Italy, pages 112-123. Morgan Kaufmann Publishers, 1998.

[10] V. Haarslev, C. Lutz, and R. Möller. A description logic with concrete domains and a role-forming predicate operator. Journal of Logic and Computation, 9(3):351-384, 1999.

[11] I. Horrocks and U. Sattler. Optimised Reasoning for SHIQ. In F. van Harmelen, editor, Proceedings of the 15th European Conference on Artifi cial Intelligence (ECAI 2002), Lyon, France, pages 277-281. IOS Press, 2002.

[12] I. Horrocks, U. Sattler, and S. Tobies. Practical Reasoning for Expressive Description Logics. In H. Ganziger, D. McAllester, and A. Voronkov, editors, Logic for Programming and Automated Reasoning, 6th Int. Conference LPAR'99, Tbilissi, Georgia, LNAI 1705, pages 161-180. Springer, 1999.

[13] F. Le Ber and L. Mangelinck. A formal representation of landscape spatial patterns to analyze satellite images. $A I$ Applications, Natural Resources, Agriculture, and Environmental Science, 12(1-3):51-59, 1998.

[14] F. Le Ber, L. Mangelinck, and A. Napoli. Design and comparison of lattices of topological relations for spatial representation and reasoning. INRIA Research Report RR-4321, 2001. 57 pages.
[15] F. Le Ber and A. Napoli. Design and comparison of lattices of topological relations based on Galois lattice theory. In Proceedings of the Eighth International Conference on Principles of Knowledge Representation and Reasoning (KR2002), Toulouse, France, pages 37-46. Morgan Kaufmann Publishers, 2002.

[16] F. Le Ber and A. Napoli. The design of an object-based system for representing and classifying spatial structures and relations. To appear in Journal of Universal Computer Science. Special Issue on Spatial and Temporal Reasoning.

[17] C. Lutz and U. Sattler. Mary likes all cats. In F. Baader and U. Sattler, editors, 2000 International Workshop on Description Logics, Aachen, Germany, pages 213-226. Aachener Beiträge zur Informatik, 2000.

[18] R. MacGregor. Representing reifi ed relations in Loom. Journal of Experimental \& Theoretical Artificial Intelligence, 5(2\&3):179-183, 1993.

[19] R. MacGregor and D. Brill. Recognition Algorithms for the Loom Classifi er. In Proceedings of AAAI'92, San Jose, USA, pages 774-779, 1992.

[20] G. Masini, A. Napoli, D. Colnet, D. Léonard, and K. Tombre. Object-Oriented Languages. Academic Press, London, 1991.

[21] R. Möller and M. Wessel. Terminological Default Reasoning about spatial information: A First Step. In C. Freksa and D. M. Mark, editors, Spatial Information Theory, Cognitive and Computational Foundations of Geographic Information Science, Int. Conference COSIT'99, Stade, Germany, LNCS 1661, pages 189-204. Springer, 1999.

[22] D. A. Randell and A. G. Cohn. Exploiting Lattices in a Theory of Space and Time. Computers Math. Applic., 23(69):459-476, 1992.

[23] D. A. Randell, Z. Cui, and A. G. Cohn. A Spatial Logic based on Regions and Connection. In 3rd International Conference on Principles of Knowledge Representation and Reasoning, pages 165-176. Morgan Kaufmann Publishers, 1992.

[24] J. Rumbaugh. Relations as Semantic Constructs in an Object-Oriented Language. In Proceedings of the 2nd OOPSLA, Orlando, USA, pages 466-481, 1987. ACM SIGPLAN Notices 22(12).

[25] T. Russ, R. MacGregor, B. Salemi, K. Price, and R. Nevatia. VEIL: Combining Semantic Knowledge with Image Understanding. In ARPA Image Understanding Workshop, 1996.

[26] A. Schaerf. Reasoning with individuals in concept languages. Data \& Knowledge Engineering, 13:141-176, 1994.

[27] M. Schmidt-Schauß and G. Smolka. Attributive concept descriptions with complements. Artifi cial Intelligence, 48(1):1-26, 1991.

[28] S. Tobies. The Complexity of Reasoning with Cardinality Restrictions and Nominals in Expressive Description Logics. Journal of Artifi cial Intelligence Research, 12:199-217, 2000.

[29] L. Vieu. Spatial Representation and Reasoning in Artifi cial Intelligence. In O. Stock, editor, Spatial and Temporal Reasoning, pages 5-42. Kluwer Academic Publishers, 1997. 